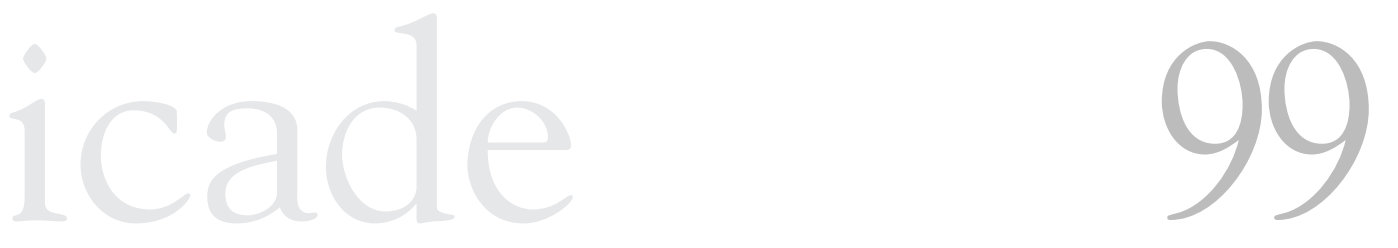

SEPTIEMBRE | DICIEMBRE [2016]

Miscelánea 



\title{
LA RENTA BÁSICA EN LA AGENDA DE PODEMOS: ¿CUESTIÓN DE VIABILIDAD POLÍTICA O DE VIABILIDAD FINANCIERA?
}

\author{
Autores: Borja Barragué Calvo ${ }^{1}$ \\ César Martínez Sánchez² \\ Facultad de Derecho \\ Universidad Autónoma de Madrid
}

\section{Resumen}

El trabajo aborda la reciente irrupción en la agenda política de dos fenómenos: el de los partidos que tienen sus raíces en los movimientos sociales y el de la renta básica. En cuanto a lo primero, uno de los eventos políticos que más interés ha suscitado en España -quizá incluso en Europa- en los últimos tiempos ha sido la irrupción de Podemos. Su resultado en las elecciones europeas de mayo de ese año y su capacidad para transformar el descontento en intención directa de voto y, quizá, en escaños, hacen que ese interés esté justificado. En cuanto a lo segundo, una de las propuestas de política social de las que más se ha venido

\footnotetext{
${ }^{1}$ borja.barrague@uam.es

${ }^{2}$ cesar.martinez@uam.es
} 
discutiendo últimamente en España, sobre todo después de que Podemos la incorporara a su programa electoral en las elecciones al Parlamento Europeo, es la renta básica universal. El trabajo se plantea explorar el futuro de esta propuesta de reforma social en la agenda de política económica de Podemos a la luz de las preferencias de sus bases. Para ello, el artículo explota los datos de una encuesta estatal sobre la propuesta de una renta básica realizada a través de internet entre el 10 de noviembre y el 15 de diciembre de 2014 por el Círculo Podemos dedicado a la renta básica universal. Se proponen y verifican dos hipótesis en el artículo: (1) ser de izquierdas va a ser menos importante en el voto a Podemos; (2) como resultado de lo anterior, Podemos no incluyó la propuesta de renta básica universal en su programa electoral para las elecciones de diciembre de 2015.

Palabras clave: renta básica; Podemos; preferencias sobre redistribución; partidos movimiento; partidos atrapalotodo.

\title{
The Universal Basic Income on the agenda of Podemos
}

\begin{abstract}
The paper addresses the recent emergence of two phenomena on the political agenda of many OECD countries: political parties born out of social movements and the Universal Basic Income (UBI). Regarding the former, one of the most important novelties in the Spanish political context has been the emergence of Podemos. Coming from nowhere, just four months after its foundation the party became the third political force in 23 of the 40 main cities in the European elections 2014. With regards to the latter, one of the social policy proposals most discussed recently in Spain, especially after Podemos included it in the program for the European elections, has been the UBI. The paper explores the future of this proposal on the economic agenda of Podemos in the light of the preferences of its constituency. To do so the paper uses an online survey on the UBI conducted during November 10 and December 152014 by the "Circle" of Podemos on the UBI proposal. We propose and test two hypotheses in the article: (1) being left-wing is going to play a decreasingly important role in the vote for Podemos; (2) as a result of this, Podemos didn't include the UBI proposal in the party's economic program for the general elections in December 2015.
\end{abstract}

Key words: basic income; Podemos; preferences for redistribution; movement party; catch-all party.

Recibido: $11 / 07 / 2016$

Aceptado: 13/10/2016

$160-183$

icade. Revista cuatrimestral de las Facultades de Derecho y Ciencias Económicas y Empresariales, no 99 septiembre-diciembre 2016, ISSN: 1889-7045 


\section{INTRODUCCIÓN}

Este artículo fue escrito originalmente a finales de mayo de 2015, unos meses antes de las elecciones generales celebradas el 20 de diciembre de 2015. En aquel momento estábamos convencidos de que, como consecuencia de la transformación de Podemos en un catch-all party o partido "atrapalotodo" en la izquierda del vector político, la propuesta de la renta básica iba a desaparecer de la agenda económica del partido. Lo que hace un año era sólo una hipótesis, ahora es una realidad. En los programas electorales de las elecciones del 20 de diciembre de 2015 y del 26 de junio de 2016, Podemos ha sustituido la renta básica por una propuesta de renta garantizada para quienes no tienen ingresos o, teniéndolos, quedan por debajo del umbral de la pobreza³.

Aproximadamente un año después de que Podemos la eliminara de su programa, el estado del debate sobre la renta básica en España ha evolucionado en un sentido ambivalente. Por un lado, y seguramente debido en parte al referéndum suizo sobre la propuesta, la renta básica es hoy una medida razonablemente conocida por los votantes españoles. Por el otro lado, la renta básica como tal -es decir, completamente universal y plenamente incondicional- difícilmente va a formar parte del programa de ningún partido de ámbito nacional en el corto plazo.

Dicho esto, la (breve) introducción de la renta básica en la agenda de Podemos ha tenido una importante externalidad positiva para los miembros más desaventajados de la sociedad española. Mientras que antes de las elecciones al Parlamento Europeo de 2015 las políticas de lucha contra la pobreza estaban fuera de la agenda política (y mediática), ahora es fácil encontrar políticas de garantía de ingresos en todos los partidos representados en el Parlamento español, con la excepción cualificada del Partido Popular. Así:

- Ciudadanos incluye en su programa un "complemento salarial garantizado para ayudar a los trabajadores con menores ingresos a que tengan un sueldo digno", que se hace efectivo a través de una devolución -es decir, que funciona como un crédito fiscal pero sólo con un tramo positivo- ${ }^{4}$.

- El PSOE propone un "ingreso mínimo vital”, que "actúe como última red de protección social para atender a las personas que queden desamparadas [...] cuando persista la necesidad", una vez agotadas todas las prestaciones de garantía de ingresos a que se tuviera derecho ${ }^{5}$. Es decir, una renta mínima de inserción de ámbito nacional y carácter subsidiario.

\footnotetext{
${ }^{3}$ Puede consultarse el programa enlazando desde http://podemos.info/programa/

${ }^{4}$ Vid. https://www.ciudadanos-cs.org/programa-electoral

${ }^{5}$ Véase http://www.psoe.es/propuestas/politica-social/ingreso-minimo-vital/
} 
- Unidos Podemos propone un "programa de renta complementaria a los ingresos existentes para todos los ingresos por debajo del umbral de la pobreza monetaria”, focalizado a los individuos o unidades de convivencia que queden por debajo de ese umbral pero prácticamente libre de condicionamientos laborales ulteriores -es decir, cuasi incondicional pero no universal-.

En España una de las claves del rápido ascenso de Podemos es lo que sus líderes han denominado la "disrupción en el cleavage político". Los modelos explicativos del voto tradicionales han venido diciéndonos que el comportamiento electoral está influido fundamentalmente por cuestiones como la clase social, la educación, la religión, etc. ${ }^{6}$. Para los líderes de Podemos, en cambio, la fractura (cleavage) izquierda/derecha ha perdido capacidad explicativa en los nuevos sistemas de partidos que se están conformando en muchos países de la OCDE tras la crisis financiera de 2008 -es un factor explicativo del voto perteneciente a la "vieja política"-. El partido de Pablo Iglesias reclama haber cavado un nuevo cleavage en el escenario político español: el cambio frente al statu quo, la nueva política frente a la vieja política, la política pro-establishment frente a la política anti-establishment.

El fuerte ascenso después de 2008 de partidos que se reclaman anti-establishment o anti-statu quo no es exclusivo del escenario político español. Algo similar ha ocurrido en Francia con el Frente Nacional de Marine Le Pen; en Austria con el FPÖ de Norbert Hofer; en Grecia con la Syriza de Alexis Tsipras; o en Italia con el Movimiento 5 Estrellas de Beppe Grillo.

Una idea más o menos extendida entre los comentaristas políticos es que partidos como Podemos, el Movimiento 5 Estrellas y Syriza, que amenazan con transformar el sistema de partidos de los países deudores de la Eurozona, son la cristalización institucional de una demanda ciudadana dirigida a los gobiernos nacionales en favor de una democracia real. A continuación, analizaremos como Podemos ha evolucionado de un movimiento social a un partido político que aspira a obtener el poder.

\section{LA EVOLUCIÓN DE PODEMOS: DE LA PUERTA DEL SOL AL PALACIO DE LA MONCLOA}

\subsection{Podemos como partido creado a partir de un movimiento social}

La idea de los partidos movimiento se la debemos originalmente a Herbert Kitschelt. En dos artículos publicados en la segunda mitad de la década de los 80 , Kitschelt $(1986,1988)$ analiza la transformación de los movimientos sociales

\footnotetext{
${ }^{6}$ Vid. por todos Lipset y Rokkan (1967).
} 
(en adelante, MM. SS.) en partidos políticos a raíz de los sucesivos movimientos de protesta antinuclear que se generalizaron a partir de la década de los 60 . Es precisamente cuando los agentes de un movimiento social dan un paso al frente y saltan a la arena institucional cuando, según Kitschelt, nos encontramos frente a un partido movimiento.

No existe ninguna garantía de que, cuando algunos agentes del movimiento social se deciden a entrar en la arena institucional, estén tomando la decisión correcta a la vista de sus objetivos y de la capacidad que tengan para solventar problemas de acción colectiva. Esto es quizá una de las claves del escaso éxito electoral del ecologismo político en España (EQUO). En lo esencial, la decisión de pasar de movimiento social -MM. SS. contra la energía nuclear, o a favor de los derechos de los animales o de los derechos del colectivo LGTB- a partido movimiento depende de dos cosas:

a) En primer lugar, depende de las barreras a la entrada en la arena institucional creadas, fundamentalmente, aunque no sólo, por la legislación electoral -otras barreras informales son las creadas por la facilidad/dificultad del acceso a la financiación o a los medios de comunicación-. A igualdad de todo lo demás, un movimiento solo dará el paso para transformarse en partido si las barreras son lo suficientemente laxas como para que los líderes del movimiento crean que existen expectativas de un resultado electoral que les permita obtener representación parlamentaria.

b) En segundo lugar, que un movimiento dé el paso para convertirse en partido depende de que los agentes sientan que existe una parte significativa del electorado cuyos intereses no están adecuadamente representados en su sistema de partidos, es decir, una explicación basada en los cleavages o fracturas de esa sociedad. Un partido tradicional puede no querer (o atreverse a) representar los intereses de un sector de la población infrarrepresentado -los desempleados, los outsiders o los veganos- porque crea que la defensa de ese sector de la población puede entrar en colisión con las preferencias de (buena) parte de su electorado cuya pérdida sería mayor que la ganancia que esperan obtener girando hacia el nuevo electorado.

Aunque sin emplear expresamente esta categoría, los líderes de Podemos han reivindicado con frecuencia su condición de partido movimiento. Por ejemplo en el "Borrador de Principios Políticos" que firman P. Iglesias, I. Errejón, J. C. Monedero, C. Bescansa y L. Alegre se puede leer que "[e]l movimiento 15M contribuyó a articular una parte de las insatisfacciones que hasta ese momento estaban huérfanas o se vivían en forma aislada y despolitizada [...] El 15M avejentó a las élites y a las narrativas oficiales, poniendo en evidencia el agotamiento de sus consensos, de sus certezas, de los marcos con los que se distribuían las posiciones y se 
explicaba el rol de cada cual en el contrato social o se canalizaban las demandas ciudadanas" (Iglesias et al., 2014: 3). Al reivindicar el 15M, Podemos parece estar reivindicando una estructura de poder horizontal y un funcionamiento (cuasi) asambleario. Un modelo de partido abierto y que no suele contar con un mecanismo institucionalizado para la resolución de los problemas de acción colectiva.

Tres características definitorias del partido de Iglesias son que (i) surge en un momento de descontento social y descrédito de los partidos tradicionales -"la casta política corrupta" - para canalizar a la arena institucional esa indignación ciudadana; (ii) detrás del éxito electoral de Podemos en las elecciones europeas hay un cleavage generacional: la gente joven -con estudios, urbana y de clase media/media-alta- es más proclive a votar a Podemos que las generaciones mayores de 30 años; (iii) cierto euroescepticismo, o al menos un discurso muy crítico con el papel que han desempeñado las instituciones de gobierno europeas en la gestión de la crisis.

Si asumimos que Podemos es la plasmación institucional de los MM. SS. ligados al 15M, entonces este partido político debería caracterizarse por un discurso netamente de izquierda. En la próxima sección discutimos esta cuestión así como la ubicación ideológica de Podemos dentro del espectro político.

\section{2. ¿Un partido de izquierdas asambleario? La evolución de Podemos hacia un partido atrapalotodo}

El concepto de catch-all party o partido atrapalotodo lo formuló por primera vez O. Kirchheimer (1966). Dicho de forma sintética, la idea es que el crecimiento económico posterior a las dos Guerras Mundiales elevó tanto la calidad de vida de la gente que se difuminaron las barreras de clase: es decir, el crecimiento del producto social creó una gran clase media cuyas preferencias eran más o menos homogéneas, creando las condiciones para la emergencia de un nuevo tipo de partido: el partido catch-all.

De acuerdo con Kirchheimer, los rasgos principales que caracterizan a un partido atrapalotodo serían tres: (i) el predominio de consideraciones cortoplacistas sobre consideraciones de tipo ideológico; (ii) un liderazgo fuerte o carismático; y (iii) centrarse en políticas tendentes a la satisfacción de los intereses y las preferencias del votante mediano, antes que en las preferencias del obrero industrial o los electores católicos (Kirchheimer, 1966: 190). ¿Responde Podemos a estas tres características? En este artículo sostenemos que sí. Más concretamente, afirmamos que la evolución de la renta básica en la agenda de Podemos ejemplifica el "giro socialdemócrata" de Podemos con el objetivo de "atrapar" todos los votos de los electores que se autoubican entre el 1 y el 4 en la escala ideológica-siendo el 1 la extrema izquierda y el 10 la extrema derecha-. Es decir, con el objetivo de atrapar los votos de todos los electores situados en la orilla izquierda del vector ideológico. 
La decisión de Podemos de eliminar la renta básica de su agenda económica obedece a ese giro pragmático o socialdemócrata, tendente a convertirse en el partido hegemónico en el espacio del centro-izquierda -desplazándose de la (extrema) izquierda en que le situaban algunas de las propuestas incluidas en su programa para las elecciones europeas-. El Gráfico 1 muestra la distribución de votos por auto-ubicación ideológica para el conjunto de partidos españoles de ámbito nacional.

\section{Gráfico 1. Distribución del voto por autoubicación ideológica}

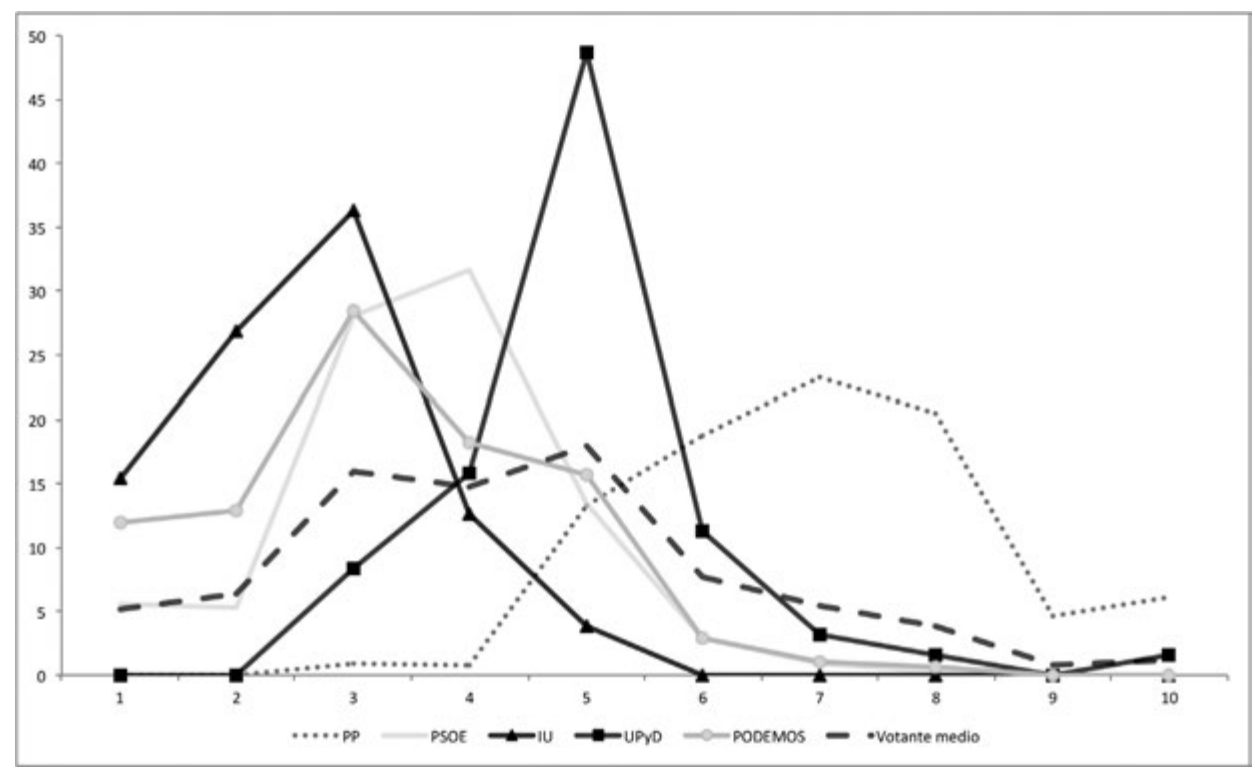

Fuente: CIS (2014a).

Como se observa en el gráfico, los simpatizantes del PP se ubican más bien a la derecha del espectro político; los de UPyD más bien en el centro; los del PSOE en el centro moderado y los de IU en la izquierda. En cuanto a Podemos, lo que vemos es que es el partido cuyo simpatizante más se aproxima al votante español medio (representado en el gráfico por la línea negra discontinua): un elector de centro-izquierda, espacio del espectro tradicionalmente dominado por el PSOE.

Pero además de que el perfil ideológico del simpatizante de Podemos es el más parecido al del votante medio, el gráfico nos dice también otra cosa: que más del 30\% del electorado se autoubica en el 4 y el 5. O dicho de otra manera: para ganar unas elecciones en España es necesario atraer a (parte de) ese electorado. Iglesias lo decía así en un entrevista publicada en mayo de 2014: "Lo que 
decimos, que quizá implique matices con respecto a lo que dicen los compañeros de IU, es que de alguna manera hay una gente que se queda fuera de los discursos tradicionales de la izquierda; que la izquierda, mientras asuma que las etiquetas ideológicas, que nuestras señas de identidad, son lo que nos definen para hacer política, tenemos un techo. Un techo que puede ser el 10, el 12, el 15, o el 17\%, y es necesario manejar un lenguaje político que de alguna manera se vincule con la mayoría social que existe"7. Lo que, según Iglesias, separa a Podemos de Izquierda Unida es que aspira a atrapar no sólo al votante de izquierdas, sino también al de centro y centro izquierda. El siguiente gráfico muestra la composición del voto a cada partido por autoubicación ideológica: hemos agrupado los valores 1-2 dentro de lo que podríamos llamar "extrema izquierda"; los valores 3-4 en lo que podríamos llamar "centro-izquierda" o "centro moderado"; los valores 5-6 que representan el "centro" -el valor medio es 5,5, de hecho-; los valores 7-8 que conforman el "centro-derecha" o "derecha moderada" y los valores 9-10 para la "extrema derecha".

\section{Gráfico 2. Composición del voto a cada partido por autoubicación ideológica}

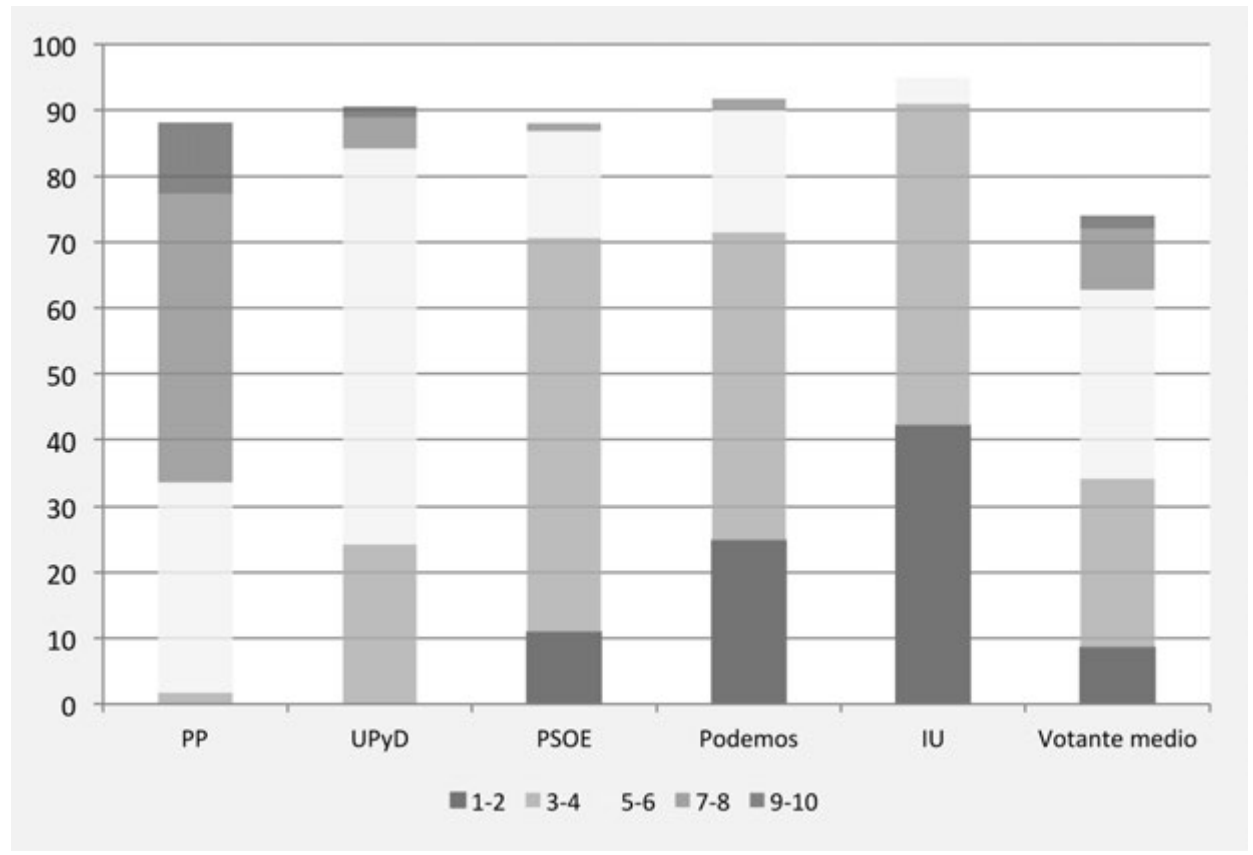

Fuente: CIS (2014a).

${ }^{7}$ http://lahiedra.info/entrevista-a-pablo-iglesias-para-poder-ganar-hace-falta-convertirse-en-pueblo/ 
Como se ve en el gráfico -que está ordenado de menor a mayor composición de electorado de izquierdas en cada partido-, Podemos se ubica en algún punto del centro izquierda entre IU y PSOE. Probablemente este hecho no sea casual. Tradicionalmente se ha venido diciendo que el votante español es de izquierda moderada. La serie histórica sobre autoubicación ideológica disponible en la web del CIS comienza en enero de 1996: el siguiente gráfico - de dos escalas verticales- resume la evolución de la autoubicación ideológica de los españoles (eje vertical izquierdo) así como la del español medio (eje vertical derecho) ${ }^{8}$.

\section{Gráfico 3. Evolución de la autoubicación ideológica y media}

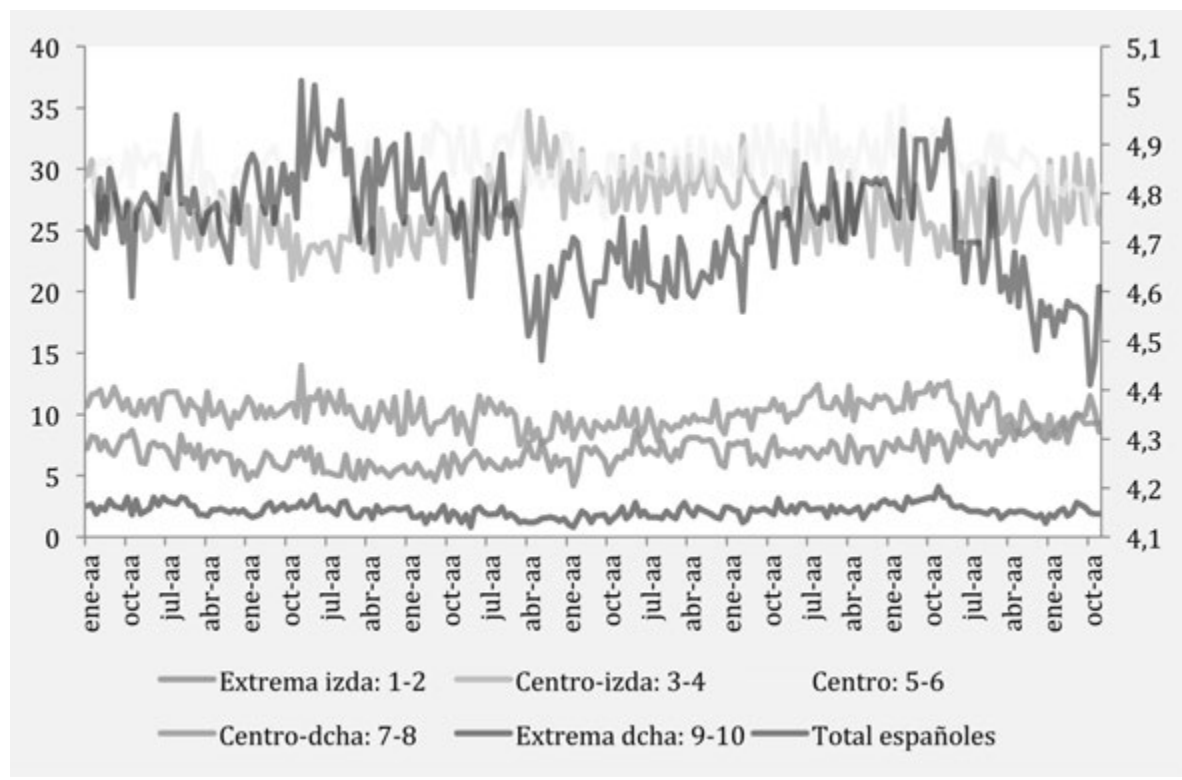

Fuente: CIS (2014a).

Del gráfico podemos extraer tres conclusiones": (1) la primera es que el porcentaje de españoles que se autoubica en la extrema izquierda es significativamente

\footnotetext{
${ }^{8}$ Pueden consultarse los datos del CIS en http://www.cis.es/cis/export/sites/default/-Archivos/Indicadores/documentos_html/sA306010040.html

${ }^{9}$ El gráfico registra al menos dos "pico de izquierdas": (i) en marzo de 2004, cuando José Luis Rodríguez Zapatero accedió al gobierno con algo más de 11 millones de votos y (ii) alrededor de mayo de 2014, cuando Podemos obtiene 5 escaños en el Parlamento de Bruselas con el 7,98\% de los votos en España; y también al menos dos "pico de derechas"; (i) alrededor de marzo de 2000, cuando el PP de José María Aznar obtiene una mayoría absoluta en la VII Legislatura española y (ii) en noviembre de 2011, cuando Mariano Rajoy se convirtió en el sexto presidente del Gobierno de España de la democracia.
} 


\section{Borja Barragué Calvo y César Martínez Sánchez}

mayor que el que se ubica en la extrema derecha y se aproxima mucho al que se declara de centro-derecha; (2) la segunda es que el español medio es, en efecto, más de izquierdas que de derechas, y que éste es un resultado que se ha mantenido estable en las dos últimas décadas ${ }^{10}$; y (3), por último, la tercera conclusión es que las elecciones se disputan en el centro/centro-izquierda antes que en el centro/ centro-derecha; en diciembre de 2014 (últimos valores disponibles), el porcentaje de españoles que se situaban en el centro y el centro-izquierda sumaban un $54,2 \%$ del electorado; la fracción de españoles que se autoubicaban en el centro y el centro-derecha era casi 20 puntos porcentuales inferior (37,9\%).

De lo anterior se desprende que, tanto si los líderes de Podemos creen efectivamente que el cleavage izquierda-derecha es una fractura del pasado como si no, el lugar que razonablemente cabe esperar que ocupe un partido como Podemos, cuyo líder ha manifestado que aspira a romper el techo de votos de la izquierda, es el centro izquierda, lugar que hasta la fecha ha ocupado el PSOE.

Cuando el 27 de noviembre de 2014 se presentó la propuesta económica elaborada por Juan Torres López y Vicenç Navarro, Iglesias afirmó que "[l]as propuestas [económicas] que asumimos son las que hasta hace no mucho tiempo iba a asumir cualquier socialdemócrata" ${ }^{11}$. ¿Cuáles son estas propuestas que enmiendan, al menos parcialmente, el programa económico con que Podemos concurrió a las elecciones europeas y acercan a Podemos a la izquierda moderada o socialdemócrata? Básicamente dos: las relativas al (im)pago de la deuda y, en lo que aquí más nos importa, las relativas a la red de protección de unos ingresos mínimos (safety net). Veamos lo que decía el programa de Podemos para las elecciones de mayo de 2014 y lo que dice la propuesta de Torres López y Navarro:

1. En lo relativo a la deuda, el programa para las elecciones europeas proponía, en su punto \$1.2, una "[a]uditoría ciudadana de la deuda pública y privada para delimitar qué partes de éstas pueden ser consideradas ilegítimas para tomar medidas contra los responsables y declarar su impago" (Podemos, 2014: 3). El documento de Torres López y Navarro no emplea el término "auditoría" -significara eso lo que significara-, ni hace alusión a deudas "ilegítimas". Todo lo que se dice ahora es que "[e]s urgente la puesta en marcha de una estrategia orientada a reestructurar y aliviar la deuda familiar", y ello porque "[e]s preciso plantear en Europa que la reestructuración de las deudas, especialmente de los países de la periferia, no es una propuesta caprichosa sino el resultado de una estrategia cooperativa que resulta mucho más favorable que la impuesta hasta ahora [...]” (Navarro y Torres López, 2014: 16-17).

${ }^{10}$ Aunque, al menos parcialmente, esto probablemente se deba a que el encuestado percibe, erróneamente, que la media es 5, cuando en realidad es 5,5 (ya que la escala comienza en el 1).

${ }^{11} \mathrm{Vid}$. http://blogs.elpais.com/cafe-steiner/2014/12/pablo-iglesias-tu-ser-socialdemocrata.html 
2. Con respecto a las propuestas para la red de garantía de unos ingresos mínimos, el cambio ha sido notable: de una renta básica universal en el programa de las elecciones europeas, a una renta mínima garantizada tal y como se explica más abajo $(\$ 3.1)$.

\section{ANÁlISIS DE LA PROPUESTA DE INTRODUCIR UNA RENTA BÁSICA EN PODEMOS}

\subsection{La renta básica y los mecanismos institucionales de Podemos}

En el programa electoral de Podemos elaborado para las elecciones al Parlamento Europeo de 2014, primeros comicios a los que se presentó esta formación política, se recoge en el punto 1.12 el "[d]erecho a una renta básica para todos y cada uno de los ciudadanos por el mero hecho de serlo y, como mínimo, del valor correspondiente al umbral de la pobreza con el fin de posibilitar un nivel de vida digno. La renta básica no reemplaza al Estado de bienestar, sino que trata de adaptarlo a la nueva realidad socio-económica. Sustitución de las prestaciones sociales condicionadas menores a la cuantía de este ingreso básico. Financiación a través de una reforma progresiva del IRPF y de la lucha contra el fraude fiscal"12.

Según consta en los propios documentos de Podemos, el programa fue elaborado en un procedimiento participativo en el que, tras la redacción de un borrador por un grupo de expertos, se fueron discutiendo adiciones y modificaciones en los denominados Círculos, que culminaron en una votación online en la que podía participar cualquier persona que se inscribiera al efecto.

La inclusión de una propuesta de establecimiento de una renta básica en el programa electoral de Podemos a las elecciones europeas llama la atención, a primera vista, por la evidente desconexión entre la iniciativa propuesta y las competencias del órgano representativo al que se concurre (Parlamento Europeo). En efecto, a pesar de los avances que han existido en los últimos años (sobre todo tras la aprobación del Tratado de Lisboa), las políticas de protección social siguen siendo competencia de los Estados miembros, por lo que la capacidad de actuación de la Unión Europea es muy limitada, toda vez que no existe en los Tratados una base jurídica específica que permita avanzar en este terreno.

No solo resulta llamativa la propuesta de una renta básica en el programa electoral de unas elecciones europeas por el hecho de que el Parlamento poco o nada pueda hacer al respecto (más allá, claro está, de declaraciones políticas sin valor jurídico), sino también por las vías de financiación que se proponen en el

\footnotetext{
${ }^{12}$ Programa disponible en http://podemos.info/wordpress/wp-content/uploads/2014/05/ProgramaPodemos.pdf
} 


\section{Borja Barragué Calvo y César Martínez Sánchez}

documento. En efecto, en el programa se establece que los recursos económicos necesarios para establecer una renta básica vendrán de la mano de una "una reforma progresiva del IRPF", así como de la lucha contra el fraude fiscal. De nuevo hay una clara discordancia entre lo que se propone y el cargo representativo al que se aspira, puesto que es evidente que el contenido del IRPF no se decide en el Parlamento Europeo sino en las Cortes.

Lo anterior pone de manifiesto que la propuesta de creación de una renta básica en el programa electoral de las elecciones europeas tenía un marcado carácter retórico, en el sentido de que se sabía que se trataba de una medida que no se iba a poder llevar a cabo en ese ámbito, en tanto que el Parlamento Europeo no tiene competencia al respecto. Asimismo, se ha de considerar que, por muy halagüeñas que fueran la expectativas de Podemos en cuanto al resultado electoral en España, el hecho de situarse fuera de los dos grandes grupos parlamentarios europeos iba a privar de capacidad de decisión efectiva a esta fuerza política. En realidad, y esto no es ni de lejos exclusivo de esta formación política, se trataba de una propuesta con marcado carácter nacional, destinada a movilizar a un electorado que, eventualmente, podría apoyarle en las próximas elecciones generales.

Meses más tarde comenzó el proceso de elaboración del programa electoral con el que Podemos concurrirá a las elecciones generales. Según han manifestado sus dirigentes, la aprobación del programa se iba a llevar a cabo a través de un sistema similar al adoptado en el caso de las elecciones europeas. Ahora bien, en este proceso existe una diferencia notable con el anterior que reside en la existencia, ahora sí, de una estructura de órganos de dirección en el seno del partido. Ahora existen dos cuerpos responsables de la aprobación de los programas electorales:

- La Asamblea Ciudadana -órgano en el que pueden participar todos los afiliados que estén al corriente del pago de sus cuotas-, responsable de "[d] eterminar la forma en la que se elaborará el programa de Podemos"13.

- El Consejo Ciudadano -órgano de dirección política elegido por sistema mayoritario-, responsable de aprobar los borradores básicos de los programas electorales.

Por más que la votación final pueda corresponder a la totalidad de los afiliados, no cabe duda de la gran influencia que van a tener los Consejos Ciudadanos, órganos de dirección política recién elegidos, en el contenido final de los programa electorales tanto por razones procedimentales (es innegable la importancia de elaborar el borrador básico), como políticas (la aprobación de un contenido contrario al definido por el Consejo Ciudadano puede simbolizar una suerte de "moción de censura"), con lo que es altamente probable que los afiliados no

\footnotetext{
${ }^{13} \mathrm{http}: / /$ podemosrivas.info/wp-content/uploads/2014/04/Estatutos-de-PODEMOS2.pdf
} 
enmienden de forma significativa las propuestas elaboradas por los representantes que acaban de elegir.

A diferencia de lo que ocurría con el programa electoral para las elecciones europeas, en el documento elaborado por Torres López y Navarro no hay referencia alguna a la "renta básica". Por el contrario, en el punto 5.4 se menciona la necesidad de aprobar un "gran Pacto estatal contra la pobreza y la exclusión social orientado a garantizar el ejercicio de los derechos humanos de contenidos económicos básicos y los que recoge la Constitución, a establecer un sistema de renta mínima garantizada". El cambio conceptual es notable, puesto que se pasa de la renta básica, incondicional y universal, a la renta mínima, destinada exclusivamente a las personas con carencia de ingresos.

La renta básica es un ingreso pagado por el Estado a cada ciudadano, con independencia de: (1) si está dispuesto (o no) a trabajar de forma remunerada en el mercado laboral; y (2) si tiene (o no) otras posibles fuentes de ingresos (Van Parijs, 1995). Una renta mínima, la fórmula por la que opta el documento de Torres López y Navarro, no es ni incondicional -el perceptor ha de estar dispuesto a aceptar una oferta de trabajo, al menos cuando se adapte a su perfil ocupacionalni universal -se establece un test de recursos, de forma que sólo son susceptibles de ser perceptores quienes acrediten ser lo suficientemente pobres-.

La no incondicionalidad tradicionalmente se ha venido justificando sobre el principio de reciprocidad y por la visión de la sociedad como una empresa común (Rawls, 2001), donde el producto social se reparte sólo entre aquellos que o contribuyan efectivamente a su creación o al menos muestren una disposición a hacerlo. Es decir, por razones de justicia: no sería justo -porque vulneraría el principio de justa reciprocidad (fair reciprocity) - repartir una parte de la tarta con aquellos que se pasan el día surfeando o fumando en el sofá de su casa viendo la televisión ${ }^{14}$.

La focalización -no universalidad- se justifica por la escasez de recursos. Desde una óptica fiscal más bien conservadora, se dirá que el Estado no puede permitirse, salvo que imponga una presión fiscal rayana en lo confiscatorio, proporcionar prestaciones y servicios a todo el mundo. Desde una perspectiva más progresista, no se termina de ver por qué no dedicar (la mayor parte de) los recursos a quienes más los necesitan -las decilas inferiores de la distribución de ingresos y riqueza-. Esto es lo que, en el ámbito de la garantía de ingresos, ha venido proponiendo tradicionalmente la socialdemocracia -que en otros ámbitos como la sanidad o la educación apuesta, al menos a priori, por el principio de universalidad en la prestación-, y es lo que recoge el documento de Torres López y Navarro. Si este giro coincide o no con las preferencias que en materia de garantía de ingresos expresan sus votantes es una cuestión que analizaremos a continuación en la última sección del trabajo (\$3.2).

\footnotetext{
${ }^{14}$ La discusión sobre si tenemos un deber moral de ser económicamente autosuficientes desborda los objetivos de este trabajo. Para una excelente discusión sobre ello, vid. Bou-Habib y Olsaretti (2004).
} 


\subsection{Preferencias de las bases de Podemos en relación con la renta básica}

El 28 de agosto de 2014 se celebró, mediante el chat de Titanpad, la primera Asamblea (virtual) de Podemos Renta Básica. Entre otros, los objetivos de Podemos Renta Básica son elaborar propuestas sobre la renta básica, su cuantía, su proceso de implantación y los recursos para llevarla a cabo, y participar activamente en el proceso constituyente de Podemos, aportando ideas a los pre-borradores sobre principios éticos, políticos y organizativos. A estos efectos, Podemos Renta Básica y el Círculo 3E -Economía, Ecología y Energía- decidieron publicar una Encuesta Estatal sobre la renta básica. La encuesta consta de 14 preguntas, se abrió online el 10 de noviembre, se cerró el 15 de diciembre de 2014 y en ella participaron 7.804 personas ${ }^{15}$. Los principales resultados de la encuesta pueden resumirse diciendo que:

1. Una mayoría muy amplia considera justa $(88 \%)$ y necesaria (86\%) la implantación de una renta básica en España, cuya cuantía sería de unos 600 700 euros $(44 \%)$ y que sólo sustituiría a las otras prestaciones sociales que sean de cuantía inferior a la renta básica (44\%).

2. Si se implantara una renta básica, la mayoría seguiría trabajando a tiempo completo $(54 \%)$ o, de no tenerlo, seguiría buscando un empleo a tiempo completo $(16 \%)$.

3. La mayoría considera que el principal obstáculo para la eventual implantación de una renta básica no es de carácter económico -un 53\% piensa que hay recursos económicos suficientes para financiarla-, sino político (58\%).

De lo anterior podríamos deducir que una mayoría muy amplia de los simpatizantes de Podemos están a favor de la implantación de una renta básica. Así, si en su programa económico la renta básica es preterida en favor de un programa de renta mínima, esto se debe a su "giro catch-all", esto es, al convencimiento de los líderes del partido de que (1) en el corto-medio plazo pueden ganar unas próximas elecciones generales y (2) para ganar unas elecciones no basta con que te voten los tuyos, sino que hay que convencer al electorado más moderado que se autoubica en el 4, 5 o incluso el 6. Esto podría apuntar a que Podemos no es un partido de funcionamiento (virtualmente) asambleario que confía la toma de decisiones políticas a la voluntad de sus bases o simpatizantes. Es decir, si Podemos se tomara en serio la idea de que "[e]n Podemos todo lo decidimos los ciudadanos y las ciudadanas" ${ }^{16}$, entonces parece que hay razones para pensar que la renta básica debería seguir formando parte de su agenda económica.

\footnotetext{
${ }^{15} \mathrm{Vid}$. Anexo.

${ }^{16}$ Como afirmaba Begoña Gutiérrez en una entrevista publicada por el diario El Mundo y que puede consultarse en http://www.elmundo.es/andalucia/2015/01/12/54b3b7ef268e3e7c228b4578.html
} 
Sin embargo, la encuesta podría contener un importante sesgo de selección, de forma que la muestra no representaría adecuadamente al conjunto de los simpatizantes de Podemos. En este caso lo que tendríamos es que: (1) la mayoría de la gente que conforma Podemos Renta Básica es favorable a la propuesta ${ }^{17} \rightarrow(2)$ Podemos Renta Básica lanza una encuesta sobre la propuesta $\rightarrow(3)$ la gente que participa en la encuesta es representativa del círculo Podemos Renta Básica-cuyos miembros son favorables a la propuesta- pero no necesariamente del total de simpatizantes del partido. No se necesitaba ninguna encuesta para saber que la gente más o menos cercana al círculo Podemos Renta Básica está a favor de la propuesta.

Sin negar que posiblemente hay algo de cierto en esta objeción, algunos datos parecen avalar sólo parcialmente la misma: (1) la cuenta en Twitter de Podemos Renta Básica tiene 838 seguidores: éstos son 6.966 menos que el número de participantes en la encuesta ${ }^{18}$; (2) el perfil en Facebook de Podemos Renta Básica le gusta a 3.175 personas: éstos son 4.629 menos que el número de participantes en la encuesta ${ }^{19}$. Aunque es muy probable que buena parte de la gente más o menos próxima al círculo Podemos Renta Básica participara, parece que su campaña de difusión de la encuesta tuvo un éxito razonable y que participó mucha otra gente aparte de la que conocía o estaba involucrada en el Círculo.

Otra posible objeción no pondría el énfasis en los problemas de selección de la muestra, sino en que ésta es superflua, puesto que es de sobra conocido que los simpatizantes de Podemos, es decir, la gente de (extrema) izquierda, tiende a ser favorable a la redistribución. Aquí conviene hacer dos precisiones: primera, la encuesta del Círculo no se refiere a cualquier tipo de redistribución, sino que se ciñe a la concreta propuesta de la renta básica. A diferencia de las prestaciones focalizadas, la universalidad característica de la renta básica hace que mucha gente de izquierdas que está a favor de la redistribución esté en contra de una renta básica - ¿Por qué pagarle una renta básica a Amancio Ortega? Eso es malgastar unos recursos que no hace falta ser economista para saber escasos"-. En segundo lugar y más importante, la encuesta no incluye ninguna pregunta que nos permita identificar el perfil de los participantes en la encuesta -situación laboral, nivel de ingresos, autoubicación ideológica, etc.-, luego es difícil saber cómo de representativos eran del votante de Podemos. Esto realmente nos plantea un problema a la hora de tratar de extraer cualquier conclusión de la encuesta. Ya que no existe aún una encuesta que nos permita identificar la relación entre variables sociodemográficas y apoyo a la renta básica, vamos a emplear los datos disponibles que más podrían aproximarse. En particular, partimos de dos hechos:

\footnotetext{
${ }^{17}$ Téngase que, de acuerdo con el acta de la primera asamblea, uno de los objetivos de Podemos Renta Básica es "[t]rabajar por la implantación de la R[enta] B[ásica] U[niversal]".

${ }^{18}$ https://twitter.com/PodemosRentaBU

${ }^{19}$ https://www.facebook.com/PodemosRentaBasica
} 
- Casi el 50\% de los votantes de Podemos se autoubican en el $3(28,43 \%)$ y el $4(18,17 \%)$.

- La renta básica es una propuesta para aumentar el tamaño del bienestar a expensas de incrementar el tamaño de los impuestos.

Teniendo lo anterior en cuenta, compararemos los resultados de la encuesta con las opiniones en cuanto a redistribución y política fiscal de los españoles que se autoubican en el centro-izquierda (3-4), según el estudio nº 3034 sobre "Opinión pública y política fiscal" publicado por el $\mathrm{CIS}^{20}$.

Un resultado conocido de la literatura sobre políticas de bienestar es que los grupos sociales que se autoubican a la izquierda del espectro ideológico son los más favorables a las políticas redistributivas -que mejoren la calidad de vida de los más pobres-, incluso si ello implica aumentar la presión fiscal. ¿Tiene esto alguna consecuencia sobre la opinión de los distintos grupos sociales acerca de la eventual expansión del Estado de bienestar en España para implantar una renta básica? El Gráfico 4 muestra lo favorables que son los españoles a la expansión del Estado de bienestar, en función de su autoubicación ideológica.

\section{Gráfico 4. Preferencias sobre el tamaño del bienestar y de los impuestos por autoubicación ideológica}

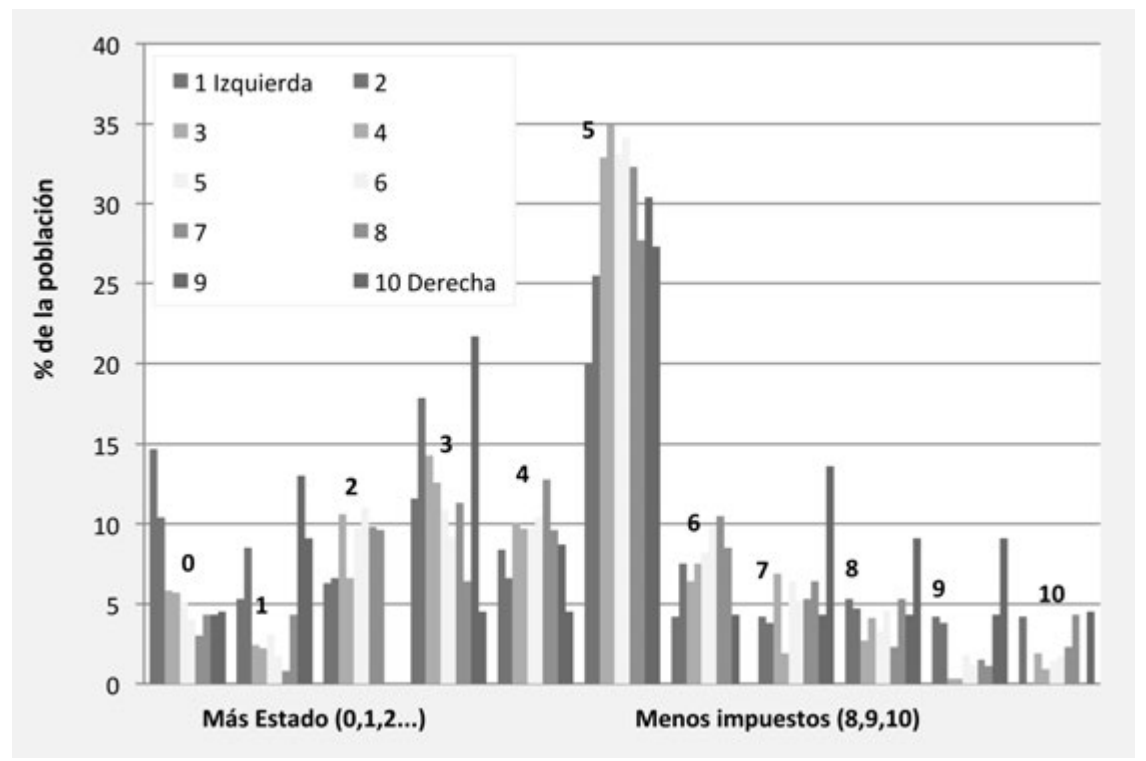

Fuente: CIS (2014b).

${ }^{20}$ Vid. CIS (2014b). 
El CIS estima las preferencias de los españoles sobre el tamaño del Estado del Bienestar y los impuestos a través de una pregunta donde el encuestado se ha de autoubicar en una escala de 0 al 10, donde 0 significa "Mejorar servicios públicos aunque haya que pagar más impuestos" y 10 "Pagar menos impuestos aunque haya que reducir servicios públicos”. El eje de abscisas del gráfico divide a los encuestados en varios grupos: los que se colocan a la izquierda del eje (los más favorables al aumento del tamaño del Estado del Bienestar y de los impuestos) y los que se colocan a la derecha (los más favorables a recortes de impuestos y de servicios de bienestar).

En el total de la muestra, un 32,2\% se autoubica en una posición intermedia, un $36,3 \%$ preferiría aumentar el tamaño del Estado del Bienestar y los impuestos y sólo un $21 \%$ preferiría menos impuestos y menos servicios y prestaciones de bienestar $^{21}$. La posición intermedia del 5 -que no indica ninguna preferencia en ninguno de los dos sentidos- es tan mayoritaria entre los encuestados que es la dominante a lo largo de todo el espectro ideológico, como se ve en el gráfico, sobre todo entre los votantes de centro y centro-izquierda.

Pero dejando a un lado esta preferencia "intermedia" mayoritaria, lo cierto es que las diferencias entre quienes se autoubican a la derecha y a la izquierda del espectro político no son muy grandes -si bien los individuos que se posicionan en el 1 tienden a posicionarse más a favor de incrementar el tamaño del Estado y quienes se ubican en el 10 tienden a preferir menos impuestos-. Como cabría esperar, quienes se declaran de izquierdas manifiestan una clara preferencia por aumentar el Estado del Bienestar, pero estos porcentajes no son tan diferentes de los de quienes se declaran de derechas: como se aprecia en el gráfico, el 21,7\% de quienes se autoubican en el 9 de la escala ideológica -extrema derecha- manifiestan una preferencia más o menos intensa por expandir el Estado del Bienestar, incluso si ello implica incrementar la presión fiscal. En España no es cierto que los individuos de izquierdas manifiesten una clara preferencia por aumentar el tamaño del Estado mientras que los de derechas prefieren recortes de impuestos; en España la posición intermedia es la dominante a lo largo de toda la escala ideológica, y en general tanto los individuos de izquierdas como de derechas prefieren aumentar el tamaño del bienestar.

Con independencia de las razones de esta "anomalía" (Fernández-Albertos y Manzano, 2012), parece haber una incongruencia entre la amplísima mayoría de los simpatizantes de Podemos que se muestran favorables a la implantación

\footnotetext{
${ }^{21}$ El cálculo se obtiene de sumar el total de la muestra que en la escala 0-10 (donde 0 significa mejorar los servicios públicos incluso si eso implica más impuestos y 10 menos impuestos, incluso si ello implica menos servicios públicos y prestaciones sociales) se autoubica en las posiciones $0-4(36,3 \%)$, el $5(32,2 \%)$ y en 6-10 (21\%).
} 
de una renta básica y las preferencias en cuanto al tamaño del Estado y de los impuestos del electorado de centro y centro-izquierda ${ }^{22}$. En efecto;

1. Por un lado, la mayoría de modelos de renta básica proponen implantar la medida a través de una reforma fiscal. Es decir, la mayoría de propuestas de renta básica implican una (gran) redistribución de ingresos desde las decilas superiores de la distribución hacia las inferiores. En el caso concreto de la última simulación hecha para el conjunto del territorio español, el modelo de Arcaróns et al. (2014) supone una redistribución de 35.000 millones de euros desde aproximadamente las 3 decilas superiores hacia las 7 inferiores.

2. Por el otro lado, en España, seguramente debido al anómalo funcionamiento del Estado de bienestar, los españoles muestran un apoyo más bien moderado a la idea de aumentar el tamaño del bienestar (la preferencia dominante a lo largo de toda la escala ideológica es 5, lo que implica algo así como “dejar las cosas como están”). ¿Cómo se explica entonces el masivo apoyo a una propuesta que implicaría (1) una reforma fiscal tendente a incrementar la recaudación y (2) una transferencia muy notable de ingresos desde los más ricos hacia los más pobres?

La explicación más plausible es que, a pesar de que el 91\% de los participantes en la encuesta respondieron que "sabía[n] ya qué era la renta básica", en realidad es muy dudoso que tuvieran el mismo concepto de renta básica en mente: esa renta básica que se distingue por su (a) incondicionalidad y (b) universalidad de los programas típicamente socialdemócratas de rentas mínimas de inserción existentes en la mayoría de los Estados de bienestar europeos. Como se muestra en la "Pregunta 4" del Anexo, sólo un 26\% de los encuestados afirmó que la renta básica "debería cobrarla la totalidad de la población (nacionales e inmigrantes acreditados)". Una mayoría (casi el 60\%) opinó que deberían cobrarla sólo aquellos que no tienen ningún ingreso en absoluto (29\%), o aquellos que "teniendo ingresos se encuentran por debajo del umbral de la pobreza" (29\%). Es decir, casi el 60\% afirmó estar a favor de una renta básica que no es una renta básica, sino una renta para pobres. Es decir, una renta mínima, que es precisamente la política de garantía de ingresos que se incluyó en el programa económico de Torres López y Navarro en sustitución de la renta básica universal del programa de Podemos para las elecciones europeas ${ }^{23}$.

\footnotetext{
${ }^{22}$ Fernández-Albertos y Manzano explican esta "anomalía española” por el peculiar mercado de trabajo español, extraordinariamente fracturado entre insiders (indefinidos, protegidos por la legislación laboral) y outsiders (temporales, precarios, con muy poca protección jurídica), que provoca que una buena parte de las políticas sociales en España -basadas en el principio contributivo y que por tanto funcionan como un seguro de garantía de ingresos en épocas de desempleo, enfermedad, accidente, etc.- sean muy poco redistributivas.

${ }^{23}$ Para un análisis de la viabilidad política de la propuesta de la renta básica, vid. De Wispelaere y Stirton (2012; 2013), o De Wispelaere y Noguera (2012).
} 


\section{CONCLUSIONES}

Este último apartado resume brevemente los principales hallazgos de nuestro estudio, que son los siguientes:

1. A pesar de que los líderes del partido afirman que Podemos ha superado el cleavage izquierda-derecha y algunos análisis politológicos lo encajan en la categoría de partido movimiento elaborada por Kitschelt, el trabajo muestra que es posible que Podemos esté más próximo a un partido catch-all de centro-izquierda que a un partido movimiento.

2. El trabajo trata de contrastar la anterior hipótesis a través de la evolución de la propuesta de introducir una renta básica en los documentos programáticos de Podemos.

3. El trabajo constata que Podemos ha abandonado la propuesta de instaurar una renta básica universal e incondicional en favor de una política de garantía de ingresos típicamente socialdemócrata como la renta mínima garantizada.

4. El trabajo analiza los datos de una encuesta que, a pesar de tener importantes limitaciones, muestra un aparente apoyo masivo a la instauración de la renta básica entre los participantes de la encuesta.

5. El análisis detallado de las respuestas muestra que, en realidad, la mayoría de los encuestados no eran partidarios de una renta básica -universal e incondicional- sino que apoyan una renta mínima para quienes carecen de ingresos. 


\section{ANEXO. ENCUESTA SOBRE UNA RENTA BÁSICA ESTATAL DEL CÍRCULO DE PODEMOS SOBRE RENTA BÁSICA; N: 7804 RESPUESTAS (SELECCIÓN DE PREGUNTAS MÁS RELEVANTES)}

Pregunta 1. ¿Le parece justo el establecimiento de una renta básica en España?

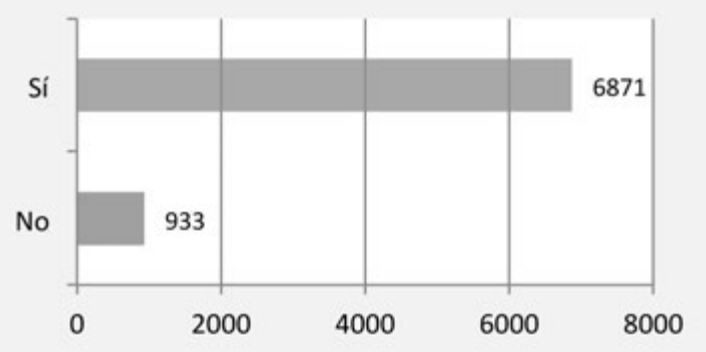

Pregunta 2. ¿Sabía ya qué era la renta básica?

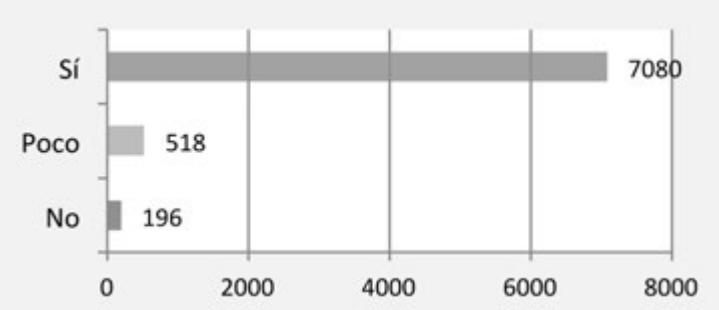

Pregunta 3. ¿Considera necesaria la implantación de una renta básica en España?

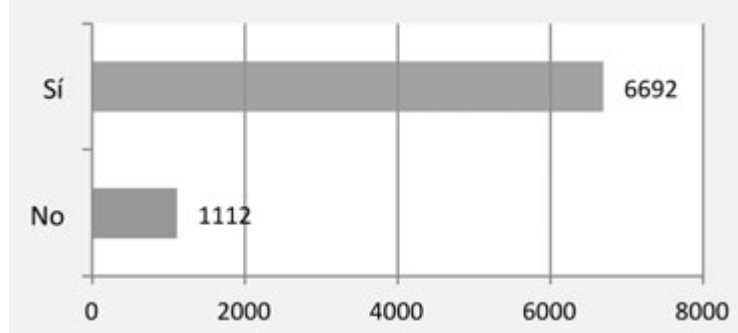


Pregunta 4. ¿Qué grupos de población considera que deberían recibir una renta básica?

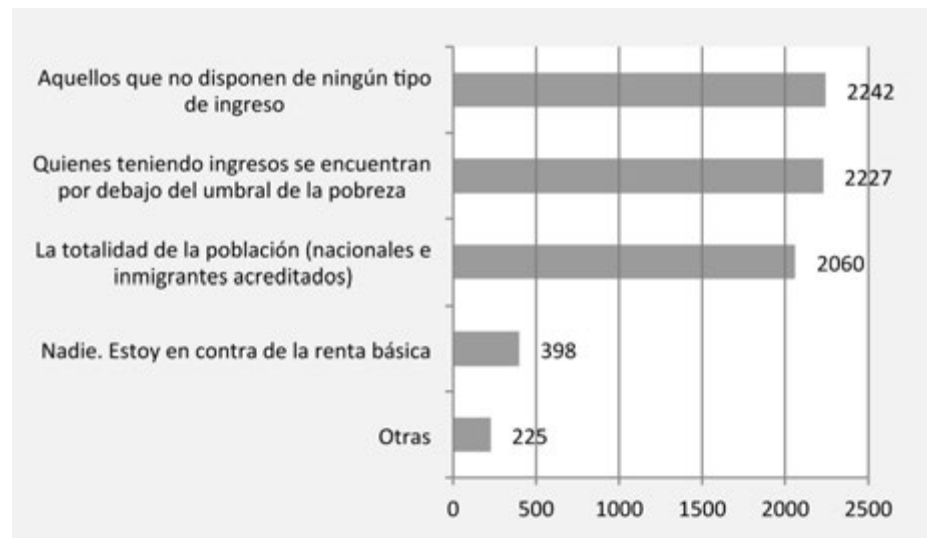

Pregunta 7. ¿Qué cantidad mínima considera necesaria para llevar una vida digna actualmente en España?

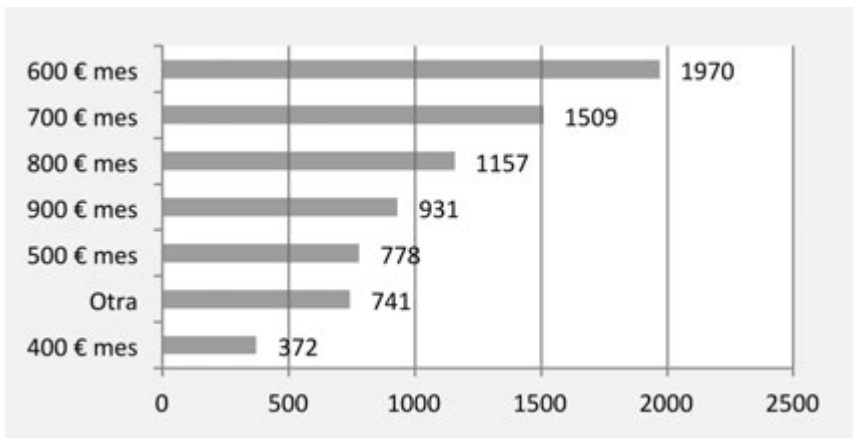


Pregunta 8. ¿Qué haría si se implantara una renta básica en relación a su actividad laboral?

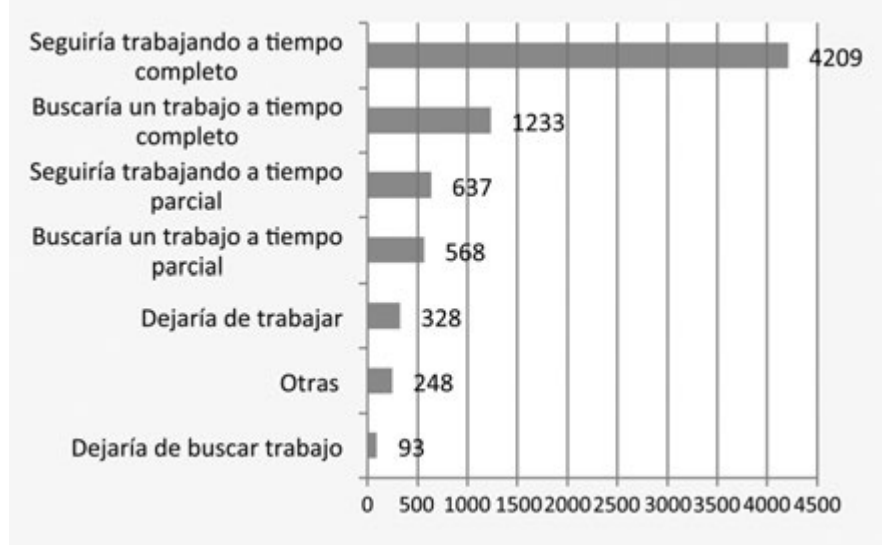

Pregunta 9. En relación a su lugar habitual de residencia

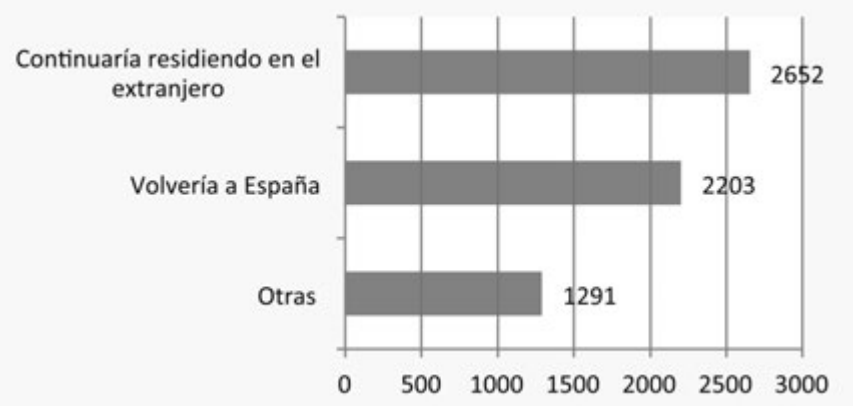

180-183 icade. Revista cuatrimestral de las Facultades de Derecho y Ciencias Económicas y Empresariales, no 99 septiembre-diciembre 2016, ISSN: 1889-7045 
Pregunta 11. ¿Considera que los menores de 18 años deben recibir una renta básica también?

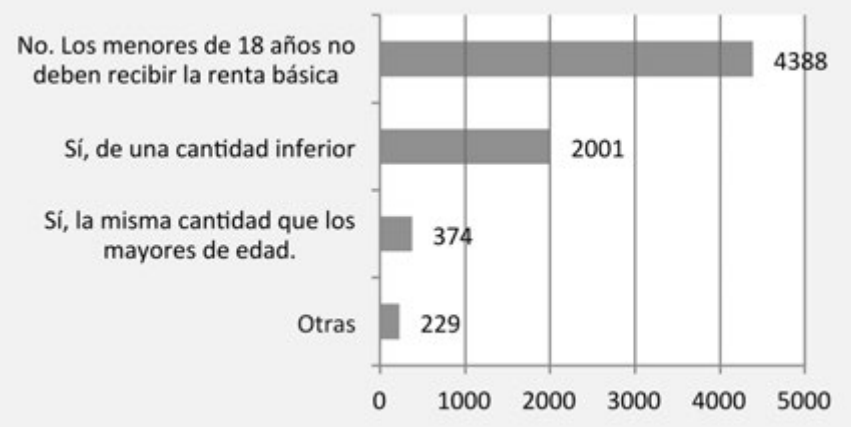

Pregunta 12. ¿La renta básica debe de sustituir a las otras ayudas sociales que el Estado otorga actualmente?

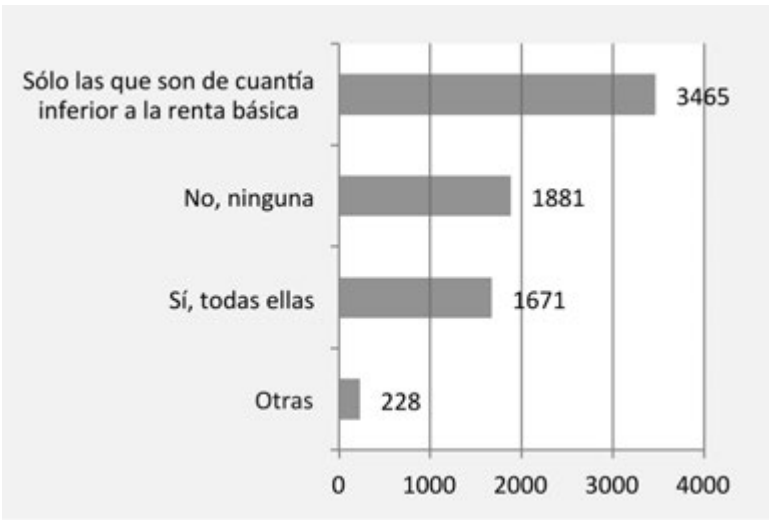

Pregunta 13. ¿Cree que hay recursos económicos suficientes para financiar una renta básica?

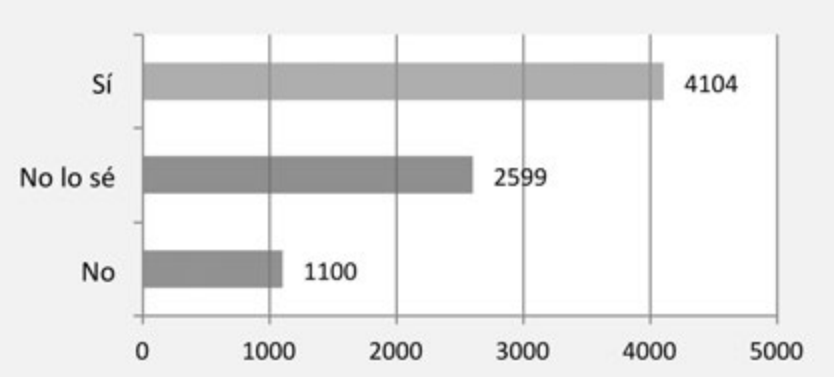


Pregunta 14. ¿Cuál piensa que es actualmente el principal impedimento para la implantación de la renta básica?

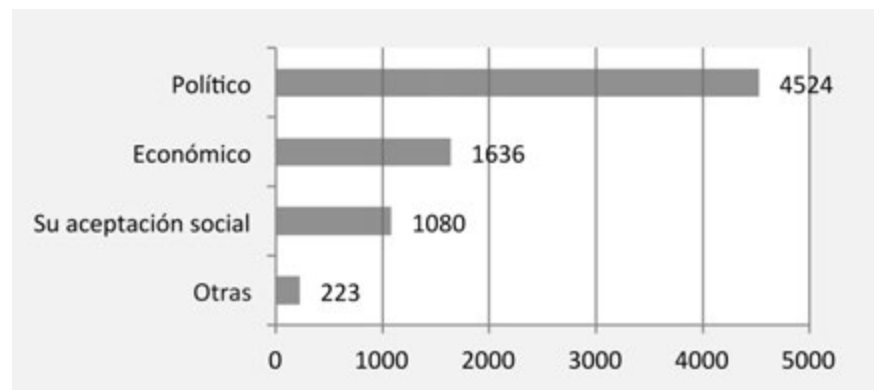

\section{BIBLIOGRAFÍA}

ARCARÓNS, J., DOMÉNECH, A., RAVENTÓS, D. y TORRENS, Ll. (2014b), "Un modelo de financiación de la Renta Básica para el conjunto del Reino de España: sí, se puede y es racional”, Sin Permiso, 7 de diciembre. Disponible online enlazando desde http://www.sinpermiso.info/textos/ index.php?id=7535

BOU-HABIB, P. y OLSARETTI, S. (2004), "Liberal Egalitarianism and Workfare", Journal of Applied Philosophy, vol. 21, núm. 3, pp. 257-270.

CIS (2014a), Barómetro (3041). Disponible online enlazando desde http:// www.cis.es/cis/opencms/ES/NoticiasNovedades/InfoCIS/2014/Documentacion_3041.html

CIS (2014b), Opinión pública y política fiscal (3034). Disponible online enlazando desde http://www.cis.es/cis/opencm/ES/1_encuestas/estudios/ver. jsp? estudio $=14100$

DE WISPELAERE, J. y NOGUERA, J. A. (2012), "On the Political Feasibility of Universal Basic Income: An Analytic Framework", en Caputo, R. K. (comp.), Basic Income Guarantee and Politics, Palgrave Macmillan, Nueva York, pp. 17-38.

DE WISPELAERE, J. y STIRTON, L. (2012), “A Disarmingly Simple Idea? Practical Bottlenecks in the Implementation of a Universal Basic Income”, International Social Security Review, vol. 65, núm. 2, pp. 103-121.

DE WISPELAERE, J. y STIRTON, L. (2013), "The Politics of Unconditional Basic Income: Bringing Bureaucracy Back In", Political Studies, vol. 61, núm. 4, pp. 915-932. 
FERNÁNDEZ-ALBERTOS, J. and MANZANO, D. (2012), ¿Quién apoya el Estado del Bienestar? Redistribución, Estado de bienestar y mercado laboral en España, Fundación Alternativas, Madrid.

IGLESIAS, P., ERREJÓN, I., MONEDERO, J. C., BESCANSA, C. y ALEGRE, L. (2014), Borrador de Principios Políticos. Disponible en http://claroquepodemos.info/wp-content/uploads/2014/09/Borrador-de-PrincipiosPol\%C3\%ADticos.pdf

KIRCHHEIMER, O. (1966), "The Transformation of the Western European party systems", en LaPalombara, J. y Weiner, M., Political Parties and Political Development, Princeton University Press, Princeton, pp. 177-210.

KITSCHELT, H. (1986), "Political Opportunity Structures and Political Protest: Anti-Nuclear Movements in Four Democracies", British Journal of Political Science, vol. 16, pp. 57-85.

KITSCHELT, H. (1988), "Organizational and Strategy of Belgian and West German Ecology Parties: A New Dynamic of Party Politics in Western Europe?" Comparative Politics, vol. 20, núm. 2, pp. 127-154.

LIPSET, S. M. y ROKKAN, S. (1967), "Cleavage structures, party systems, and voter alignments: an introduction", en Lipset, S. M. y Rokkan, S. (coords.), Party Systems and Voter Alignments: Cross-National Perspectives, Free Press, Nueva York, pp. 1-64.

NAVARRO, V. y TORRES LÓPEZ, J. (2014), Un proyecto económico para la gente, PODEMOS. Disponible en http://web-podemos.s3.amazonaws. com/wordpress/wp-content/uploads/2014/11/DocumentoEconomicoNavarroTorres.pdf

PODEMOS (2014a), Programa para las elecciones europeas. Disponible en http://podemos.info/wordpress/wp-content/uploads/2014/05/ProgramaPodemos.pdf

PODEMOS (2014b), Estatutos del partido. Disponible online en http://podemosrivas.info/wp-content/uploads/2014/04/Estatutos-de-PODEMOS2. pdf

RAWLS, J. (2001), Justice as Fairness: A Restatement (ed. Kelly, E.), Belknap Press, Cambridge.

VAN PARIJS, P. (1995), Real Freedom for All: What (if Anything) Can Justify Capitalism?, Oxford University Press, Oxford. 